\title{
Model of Learning Objects Exchange between LCMS Platforms through Intelligent Agents ${ }^{1}$ Modelo de intercambio de objetos de aprendizaje entre plataformas LCMS mediante agentes inteligentes ${ }^{2}$
}

\author{
Paulo Alonso Gaona-García ${ }^{3}$ \\ Carlos Enrique Montenegro Marín ${ }^{4}$ \\ Elvis Eduardo Gaona García
}

doi:10.11144/Javeriana.iyu 19-2.mloe

How to cite this article:

P.A. Gaona-García, C.E. Montenegro Marín, and E.E. Gaona García, "Model of learning objects exchange between LCMS platforms through intelligent agents", Ing. Unv., vol. 19, no. 2, pp. 391-413, 2015. http://dx.doi.org/10.1114/javeriana. iyul9-2.mloe

\footnotetext{
${ }^{1}$ Reception date: December $22^{\text {nd }}, 2013$. Acceptance date: March $7^{\text {th }}, 2015$. This article is the result of the investigation project named Interoperability over LCMS platforms of the GIIRA research group at Universidad Distrital Francisco José de Caldas recognized in Colciencias in A category.

${ }^{2}$ Fecha de recepción: 22 de diciembre de 2013. Fecha de aceptación: 7 de marzo de 2015. Este artículo hace parte del proyecto de investigación: Interoperabilidad web sobre plataformas LCMS, del grupo de investigación GIIRA, reconocido en Colciencias en categoría A.

${ }^{3}$ System Engineer and MSc. in Information Sciences and Communications. PhD in Information of Engineering and Knowledge, University of Alcalá, Spain. Professor at Engineering Faculty, Universidad Distrital Francisco José de Caldas, Bogotá, Colombia, and active member of GIIRA research group. E-mail: pagaonag@udistrital.edu.co

${ }^{4}$ System Engineer and MSc. in Information Sciences and Communications. PhD in Systems and Informatics Services for Internet, University of Oviedo, Spain. Professor at Engineering Faculty, Universidad Distrital Francisco José de Caldas, Bogotá, Colombia. Director of GIIRA research group. E-mail: cemontenegro@udistrital.edu.co

${ }^{5}$ Electronic Engineering and MSc. in Information Sciences and Communications. Actually is PhD (c) in Engineering. Professor at Engineering Faculty, Universidad Distrital Francisco José de Caldas, Bogotá, Colombia. Director of GITUD research group. E-mail: egaona@udistrital.edu.co
} 


\section{Abstract}

Currently, there are a variety of digital resources hosted on the Internet, based on access points such as digital libraries and repositories. The majority of strategies to store information are used by specialized documentation centers, academic institutions, and databases of regional development policies. These entities have been directing their efforts to improve the access over a collection of digital resources for academic and professional purposes. There is still a lot of educational material that is hosted on content management tools known as Learning Content Management System (LCMS); however, those tools do not perform the corresponding indexing of digital resources for the use of repositories. In fact, this requirement reduces the access to digital resources even from the inside of each academic institution, losing coverage and recognition in other learning environments. Moreover, this factor also limits the enrichment and linking of related academic material. Then, strategies such as Linked Data cannot be extensively used to share digital resources. Therefore, this article aims to devise an indexing strategy to manage digital resources hosted in different LCMS by defining services that facilitate the exchange of digital resources and their reuse. Case Study: Efront and Moodle platforms.

\section{Keywords}

indexing; reuse; learning objects; metadata; semantic web; software agents

\section{Resumen}

Actualmente existe una gran variedad de recursos digitales alojados en Internet a partir de puntos de acceso como bibliotecas digitales y repositorios. La mayoría de estas estrategias, definidas por centros de documentación especializados, instituciones académicas e iniciativas o planes de acción regionales, han estado orientando sus esfuerzos a facilitar el acceso a materiales con fines de uso académicos o profesional. Sin embargo, existe todavía una gran cantidad de materiales educativos que se encuentran alojados en herramientas de gestión de contenidos conocidas como LCMS, y que carecen de una indexación en sus recursos a partir del uso repositorios. Esta necesidad reduce su uso, exclusivamente dentro de cada institución y pierden alcance y reconocimiento en otros ambientes de aprendizaje con mayor cobertura para su reutilización. Este aislamiento también limita el enriquecimiento y vinculación de material académico relevante en un área de conocimiento específico mediante el uso de estrategias como Linked Data. Por lo tanto, el artículo se orienta a plantear una estrategia para la indexación de recursos digitales alojados en diferentes LCMS mediante la definición de servicios que faciliten el intercambio de recursos digitales y su posterior reutilización. Caso de estudio: plataformas Efront y Moodle.

\section{Palabras clave}

agentes de software; indexación; objetos de aprendizaje; LCMS; reutilización; metadatos; web semántica 


\section{Introduction}

The semantic Web is one of the initiatives that over the course of its development has endeavored to simplify the access to relevant information. To achieve this goal, the semantic Web uses enriched vocabularies and data models to describe knowledge management. This development has been supported by the collaborative effort of hundreds of groups on the Internet and organizations such as W3C, in order to improve and define the communication models that obtain the support and management of information on the Web in an efficient way. Following this approach, and under the proposal defined by the very founder of Internet, Tim Berners Lee [1], new alternatives have been raised to share and access to these relevant resources through the connection of Linked data.

The data connection approach has been widely accepted in the research community, including a broad spectrum of knowledge areas, growing from the well-known initiatives such as DBpedia [2], to the version of Wikipedia, which connects thousands of digital resources on Internet, and including the Open Discovery Space [3], [4], an European Union project to connect educational resources to support European academic institutions. A similar project associated to these alternatives is called Europeana [5], this proposal is focused on the compilation of the largest quantity of digital resources related to European cultural heritage defined by thousands of specialized content providers. Due to the release of this initiative to share and publish several digital resource descriptions, as defined from its metadata, Europeana collects one of the major content suppliers in European cultural heritage [6], which establishes it as one of the larger initiatives to share and reuse digital resources for educational purposes. All these proposals have in common the use of learning objects and their connection through Linked data. This initiative improves the searching process from specialized vocabulary and enriched languages to Simple Knowledge Organization Scheme (SKOS) [7], [8]. 
Another useful strategy to manage educational material is based on the use of open source Learning Management Systems also known as Learning Content Management System (LCMS). These tools enable three characteristics: (1) The management of the learning process; (2) the storage of contents and educational material; and (3) the development of strategies to improve complementary training. Nevertheless, the restrictions to share information between diverse platforms (i.e., constrains to access to material, among others) have conditioned their potential to simply relevant educational material linking and the allocation of resources for a large academic community.

Additionally, LCMS have been used in platforms oriented to support learning management through the creation of courses to share material and accomplish complementary training. However, most of this stored material is not continually updated and the reuse of information is a difficult process in learning environments. This phenomenon reduces the quality of material reuse. Usually, the material is stored in various platforms through numerous factors that reduce their scope and subsequent impact in the academic environment. Some of these factors are: (1) A lack of knowledge about tools to create and manage learning objects; (2) the absence of institutional policies towards management and updating of material for reuse; (3) the lack of initiatives to create digital repositories to index educational material within institutions; (4) low usage of resources; (5) few search strategies to facilitate access to digital resources (learning description models can help to facilitate the search processes); and (6) difficulties in the usability associated to search interfaces [9]-[11].

Associated with the use and characteristics of digital resources to simplify reuse, a number of descriptions are required to enable a major scope and visibility. These strategies had covered the metadata definition, which came from an exchange data model such as LOM [12] or a specification model such as SCORM [13], supplying a digital resource description. Because of these processes, activities aimed to create learning objects are released to encourage searching processes and learning object localization [14]-[16].

Using this insight, the goal of this article is to present a proposal to enable the search and exchange of learning objects process between two LCMS platforms (Efront and Moodle), with the purpose of locating relevant learning objects for subsequent bonding with initiatives such as Linked Data, over an area of greater knowledge. To achieve this objective, section 1 describes relevant concepts to develop this proposal. In section 2 the methodology to develop the search strategies is presented. Section 3 describes: (i) An implemented search 
web based on SCORM content and, (ii) the development of two modules to perform the interoperability between the two LCMS platforms based on different architectures. In section 4 an evaluation of the developed agents is presented. Section 5 presents results and analysis. Subsequently we present a section with the discussion, conclusions, and future work.

\section{Conceptual Framework}

Information exchange and interoperability between platforms have been defined in further education areas such as Geographic Information System GIS [17]. Moreover, diverse strategies to collect and connect digital resources are carried out through well-known tasks, such as harvesting. This process is also known as learning objects indexing; it enables the association of thousands of digital resources created and stored in digital repositories through the Open Archives Initiative Protocol for Metadata Harvesting (OAI/PMH) protocols [18], [19]. The aim of this proposal is based on the reuse of a digital resource and its adjustment within a learning environment [18], [19]. Likewise, the OAI/PMH protocol is seeking to improve academic material research stored in databases or external repositories which are not connected in conventional searching engines such as Google and Yahoo [20]. This type of activities have enabled the definition of specifications and policies to use and share digital resources from metadata meaning [21], [22]; improving processes to create educational material by a large user community with emphasis on searching educational material for its reuse. Nevertheless, it still occurs that this material is stored in diverse open source learning management platforms (Learning Content Management System [LCMS]), which represent a great opportunity for material reuse in academic networks, and also it makes possible to develop an extended coverage and visibility of academic material.

Currently, the studies about human knowledge and its depiction in computation are still based on a centralized and widespread artificial intelligence meaning [23]. To solve great problems, a major knowledge is required and this knowledge has to be represented in some manner through defined languages using a computer. To adjust this problem, applications are designed to define how knowledge can be described [24]. An example of these applications is software agents: software designed to accomplish a particular activity and act taking certain rules or designed patterns from a describing knowledge schema [25]. Accordingly, knowledge can be used by a software agent describing specific representations. 


\subsection{Software Agents}

A software agent is defined as any system able to perceive environment changes [25], [26]. Therefore, a software agent perceives information about its current state and compares it with its objectives and finally acts based on the rules and gained experiences within an environment. Agents operates in context, in other words, they do not usually perform the same tasks and even do not follow similar ways to accomplish a specific task.

Also, an agent has the authority to decide when it is appropriated to complete a task. They do not need to be invoked; in fact, they have to be active all time. Agents maintain modularity properties, low coupling, reliability, efficiency, persistence, among other, to accomplish their objectives [27].

\subsection{Web Services}

A web service is a set of applications that are related on the Web with other technologies to exchange data in order to deliver services to users through the interoperability between them. The users can access a service from a remote site through any provider that offers a network platform. Service providers deliver communication mechanisms to interact between them to represent information dynamically, these mechanism assume that the architecture makes the interoperability and extensibility possible among other applications to perform a complex action [28].

A web service allows to share information and also it allows sharing functions regardless the platform, due to enriched languages such as XML. The latter makes possible to link the web services with others services to achieve new tasks. In this proposal, during the exchange process activities a number of services must be specifiedas to guarantee quality interoperability between the LCMS selected, even though each service relies on different platforms or standard frameworks. In this respect, it is taken as a basis the architecture defined by WC3 [29], the components of which are shown in Figure 1.

\section{Methodology}

In order to carry out this project strategy, an application needs at least one LCMS platform to connect all contents. In fact, a fully completed connection between two or more LCMS platforms produces an environment of interoperability between platforms to share content through web service definitions. Due to this fact, the application executes searching processes in accordance with user needs also aligned up with an educational institution project. This connection is made through a module to enable authentication and later access to services for each user. 
Figure 1. Web service architecture defined by W3C

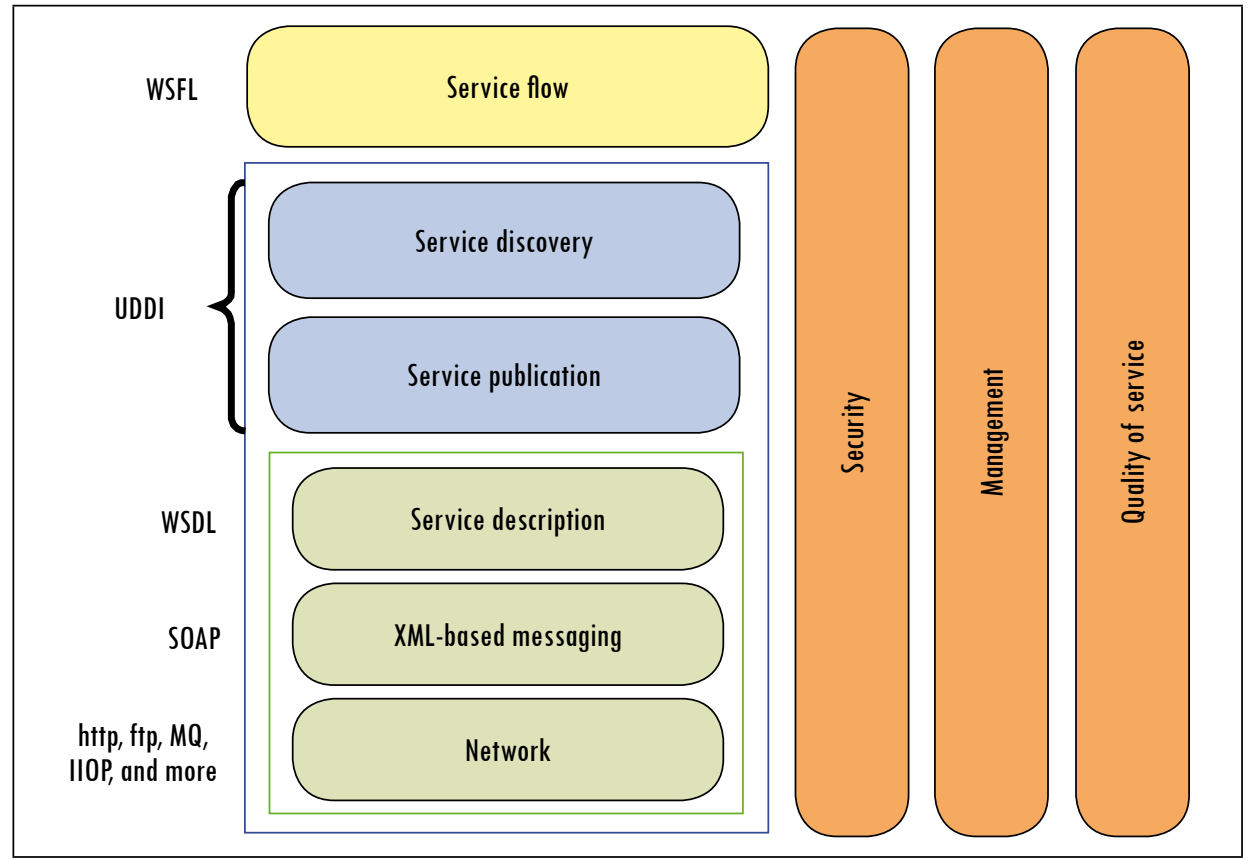

Source: [29].

\subsection{Selection Criteria for LCMS Platforms}

The criteria of selection of Efront and Moodle were given by: (1) Their modular developed structure based on dynamic learning, object-oriented, and modular environment; (2) the platforms developed in PHP language enable operating system execution due to their supporting convenience in a Web server such as Apache; (3) they are distributed by an Open Source under GNU public license; and (4) they have a compatibly standard with SCORM version [13] and with the IMS packaging since 2006 [30].

\subsection{Validation Method Definition}

The proposal pretends to enable the search of learning content based on SCORM specifications, and also to share content between two different LCMS platforms. The specification has been defined due to its easy adaptation and application over most projects, with emphasis in the base developing and learning objects creation in a structured manner. In order to perform this activity, the proposal was designed to deploy a module to ensure interoperability between the selected platforms (Efront and Moodle) and to guarantee its functionality. The module is 
compose by three Web services in the same programming language for Efront and Moodle respectively:

1. To authenticate registered users in the platform, who can access to the search engine and download SCORM content.

2. To entrust the described metadata operation in each learning object. The process would allow to store and index context with the aim of enabling future searches defined by the user criteria.

3. The last developed web service goal consists of developing codification and packaging processes in order to share relevant content selected by users.

For the purpose of integration of a LCMS platform, a communication module is necessary to set up a LCMS platform; which means, the information requires to be adjusted to provide three essentials Web services for browser functionality. Therefore, the Web services defined to be used by LMCS are: (1) Authentication (to allow users access to the browser platform); (2) search and metadata access (to obtain metadata by SCORM content in the platform); and (3) connection of learning objects to services (to associate contents accordingly to search criteria defined by users). Figure 2 presents a description of this process.

Figure 2. Interoperability module defined for LCMS platforms

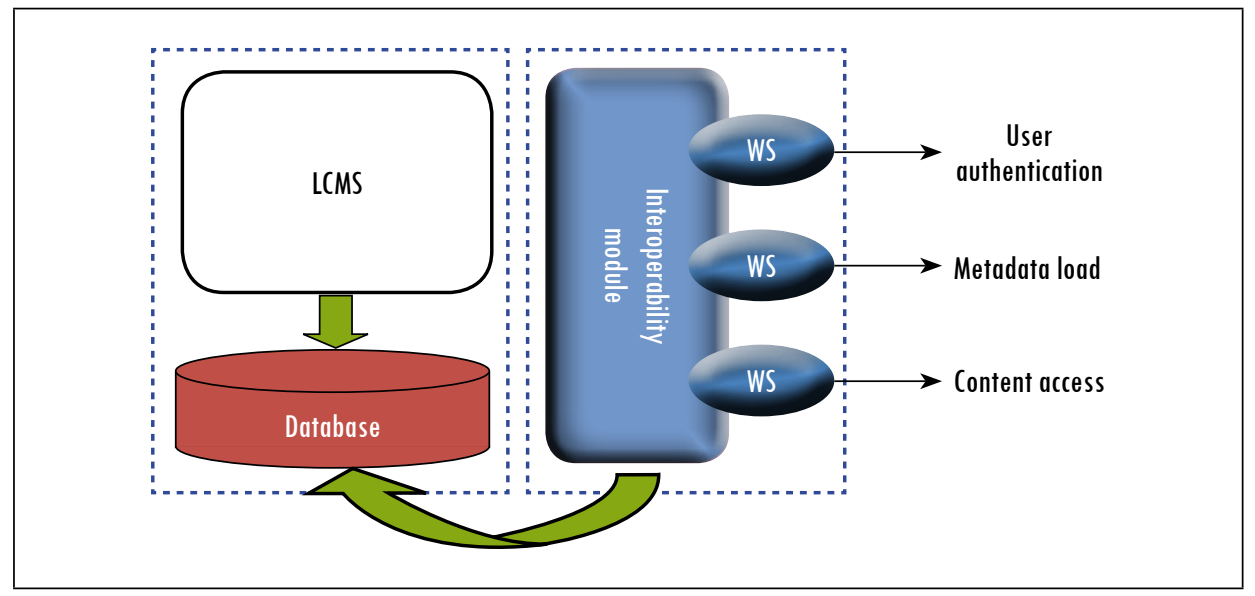

Source: authors' own elaboration

Once the module is set up in the platform, the clients are defined in each defined Web service. Then, they are added to the browser to connect contents 
to the new platform, to subsequently link them to the browser. Figure 3 shows the process in detail.

Figure 3. Platform integration work flow

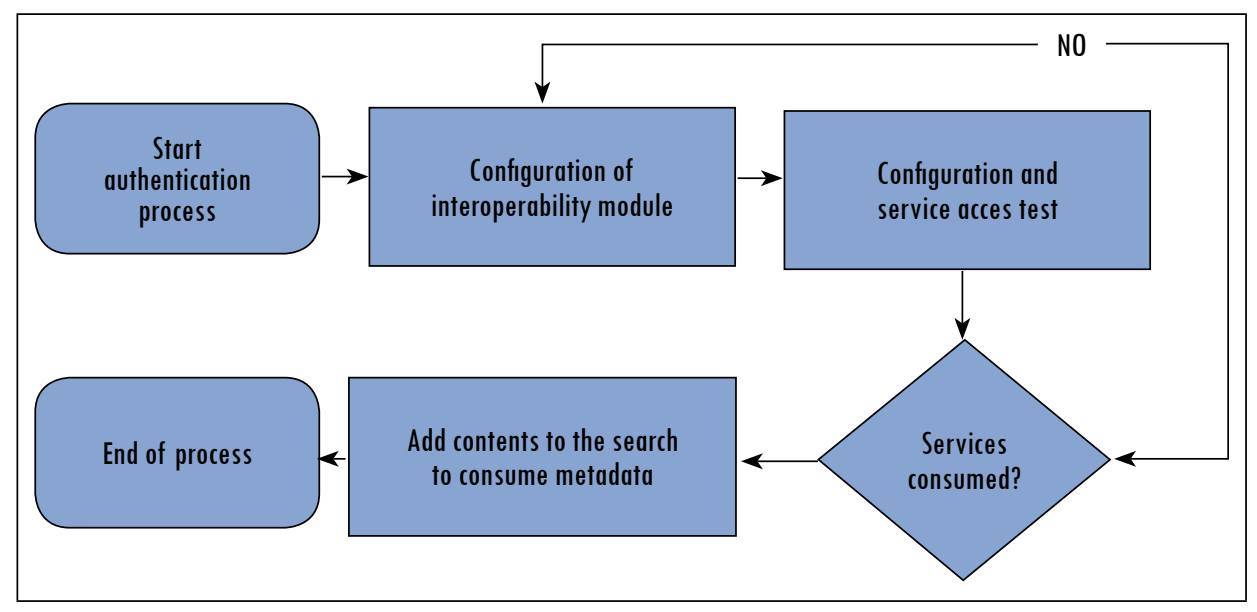

Source: authors' own elaboration

\subsection{Access Service Process}

Services provided by these platforms are consumed on different execution moments. The metadata service is consumed before the application starts running; it needs to access metadata content in each connected platform before starting a search process. After one access, this service carries out the organization and indexing process for the information agent, they have to check if all elements needed to make its job are active. On the other hand, the other two services (authentication and download) are consumed during execution time; one during the validation moment and the other one during the download.

The last two services are independent, called by each user during their interaction with the browser as shown in Figure 4.

A process to carry out search through the interaction between users in the web application is defined below. In this scenario three actors are defined: the user who makes the search, the search agent, and the Gateway agent who receives a user petition a sends it to the search agent for each request made by the user application. The work flow of this process is defined as:

- The search criteria are defined by the user accordingly to key words related to the learning object within the browser interface. 
Figure 4. Connection between the work flow of learning bbjects

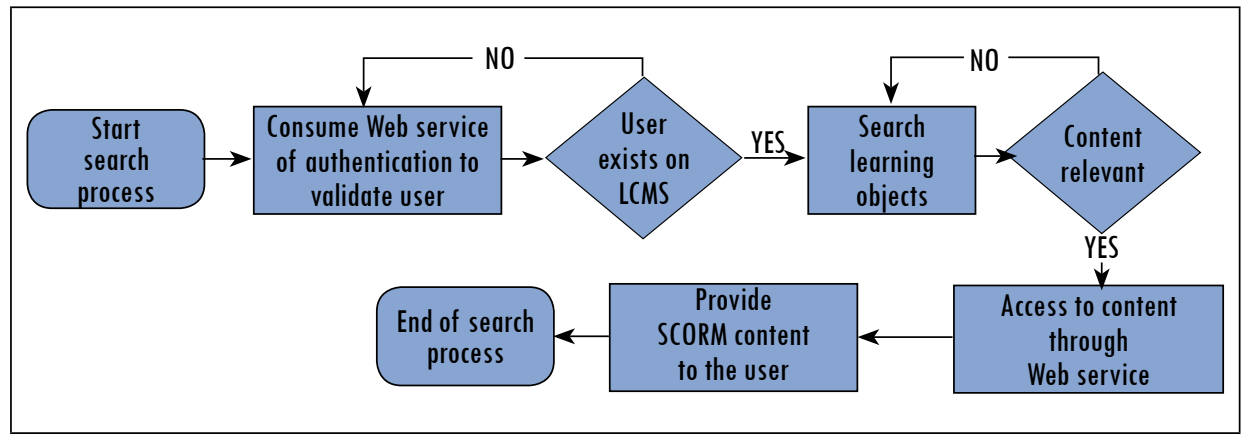

Source: authors' own elaboration

- These words are received by the Gateway agent, which is responsible for the communication of user needs with the search agent.

- The search agent develops the selection of contents based on the information provided by the gateway agent and the metadata registered in the system.

- The contents are organized based on relevance and coincidence in the metadata browser.

- The search agent returns to the gateway agent with a coherent list of content according with the related metadata information.

- The gateway agent organizes the diverse results and its corresponding information.

- Finally, each user can access to content that he considers convenient according to their needs.

In the search process the agent receives a petition to encounter learning content related with the information that the user had considered convenient. The agent compares this information with metadata contents that are indexed and divided by their characteristics for easy organization and filtering. The next section describes the working model defined for the module implementation and authentication, including connection and access to learning objects stored in LCMS platforms.

\section{Working Model Planning}

The description of each module defined to carry out authentication, validation, and search learning object in each selected LCMS platform is presented below. 


\subsection{Authentication Module}

The search of learning objects are based on SCORM upon diverse virtual learning platforms (LCMS). Hence, a browser is defined to integrate custom content to each LCMS platform through metadata described in SCORM package. Consequently, one of the objectives of this project is to share isolated content in diverse educational institutions which use LCMS platforms as a learning tool.

In order to establish the trust and reliability of the contents, a security layer is proposed under the Web of Trust content [31], [32], where users keep a certain degree of trust with small group of users. This reliability principle enables the user to be authenticated in an educational institution to share resources in a secure manner, in such a way, that the user can have access to contents of all institutions linked to the prototype, as it is presented in Figure 5.

Figure 5. Authentication module between different platforms in rducational institutions

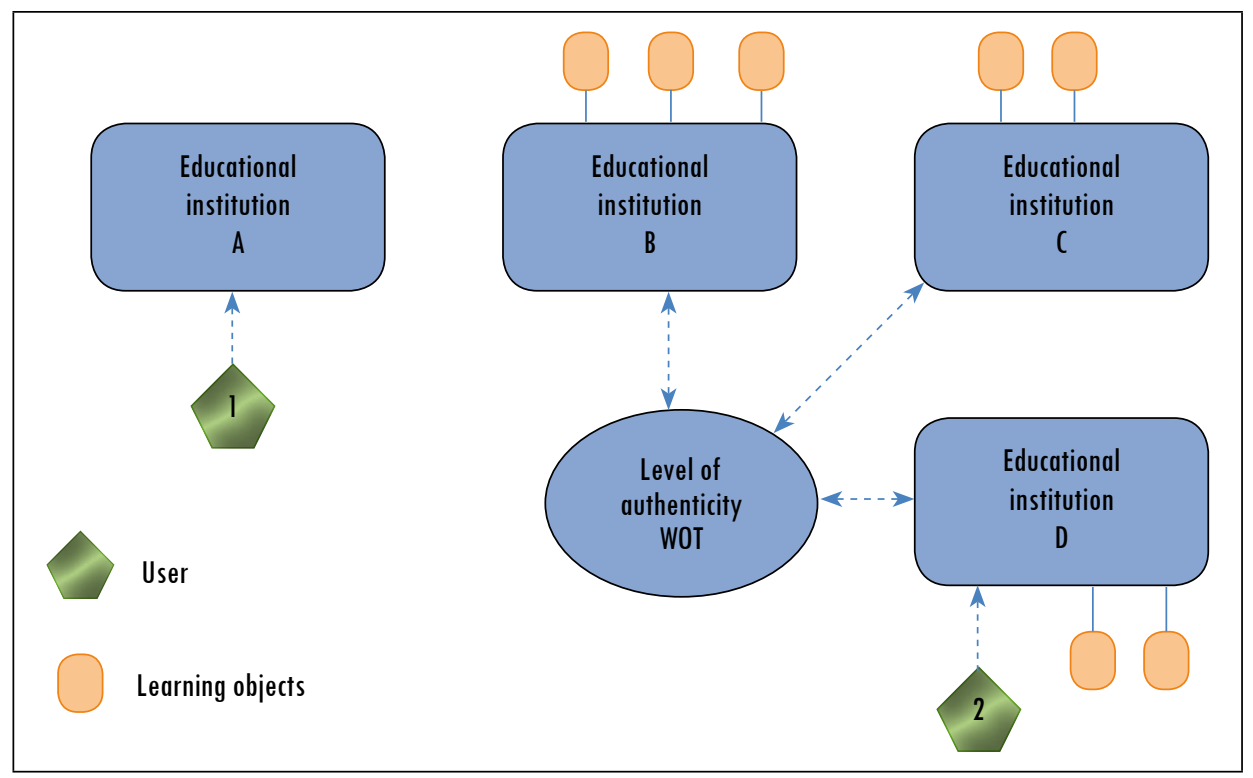

Source: authors' own elaboration

Figure 5 shows an example of this authentication model, generating a secure work environment in diverse institutions under the Web of Trust (WOT) concept. The definition of trust levels in LCMS platforms has been defined in a previous work [33]; it enables the sharing of learning objects, promoting also the development and updating of learning objects in a virtual learning environment starting 
in academic communication networks. This process would allow the search and access to reliable contents on Internet.

When learning objects are supported by educational institutions, these provide a large degree of trust to the truthfulness of the content, due to fact that they are frequently used into a learning process. The project is based on this foundation to offer an environment where users can locate the content according to their needs, under trust principles, and access resources in a relevant manner.

In order to develop an authenticity level in various LCMS platforms, an ontological model is defined to represent trust levels, specifically within a LCMS platform using OWL-DL created by the W3C Web Ontology (Web Ontology) Working Group. Methontology [34], [35] was the developed methodology, which enables the construction of ontologies at the knowledge level, and it has been proposed by the Foundation for Intelligent Physical Agents (FIPA), which promotes interoperability through applications based on agents [36]. Figure 6 shows the ontological model proposed to define security levels, as proposed by [37].

Figure 6. Ontological model to define trust levels

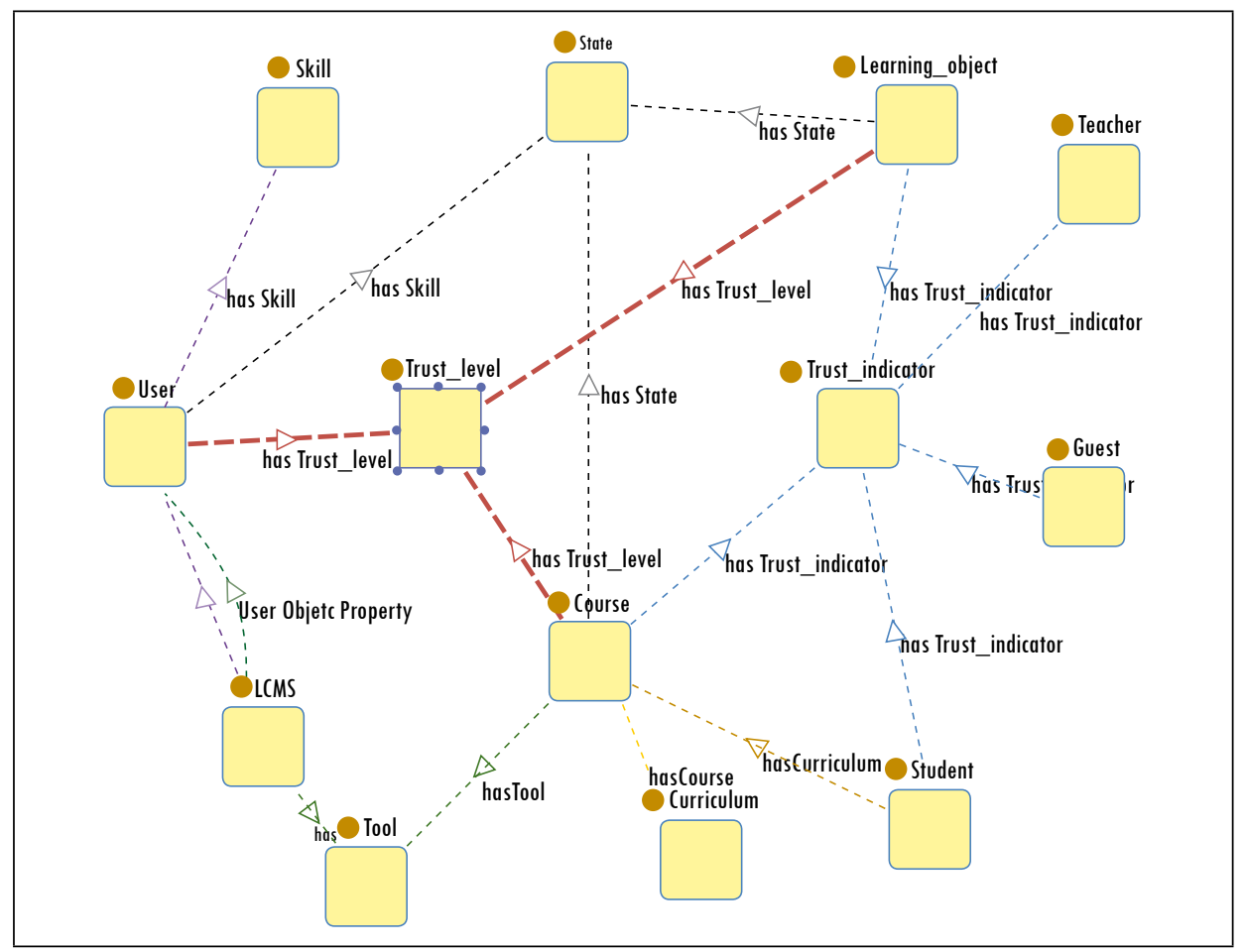

Source: authors' own elaboration 
Hence, the software agent attends the search of learning objects in the storage of LCMS platforms. This agent uses the ontological model described above, in Figure 6, to establish a communication link; the users employ it to perform search processes. Two basic processes are executed by the agent: the first process is based on the search, and the second one is based on results organization.

During the search process, the agent receives a petition to find learning contents related with the information that users considered convenient. The agent compares this information with the metadata contents that are indexed and divided by their characteristics to enable the organization and filtering of data.

\subsection{Connection Module}

The connection process of resources define the sequence from an educational institution to the authentication module through the LCMS platform. These processes allow to share trusted content and access content from other institutions.

The development environment relies on the LCMS platforms to supply SCORM contents to share. Hence, the goal of this proposal is based on the integration and inter-operability between platforms to supply a search process. Bearing this in mind, the system is divided into various sections: First, the section associated to the search core related to the metadata logic, indexing, searching, and content results. The second section is focused on inter-operability between platforms, through web services to access information and connect learning objects without modifying contents or platform functions.

\subsection{Communication Module}

For each LCMS platforms (E-Lera and Moodle) a module responsible for communications with the browser without modify normal functionality within each LCMS has been defined. This is a specific module in each platform, according with its architecture, which enables it to be set up according with the connection needs. In Figure 7 the application structure is shown.

For this module, the browser obtains information and provides interoperability content services through related searches based on metadata descriptions defined in each learning object. Therefore, each user can directly access the search results and access learning objects in a transparent manner on each LCMS platform where they are hosted, using a transparent communication process to access the contents. 
Figure 7. Application structure

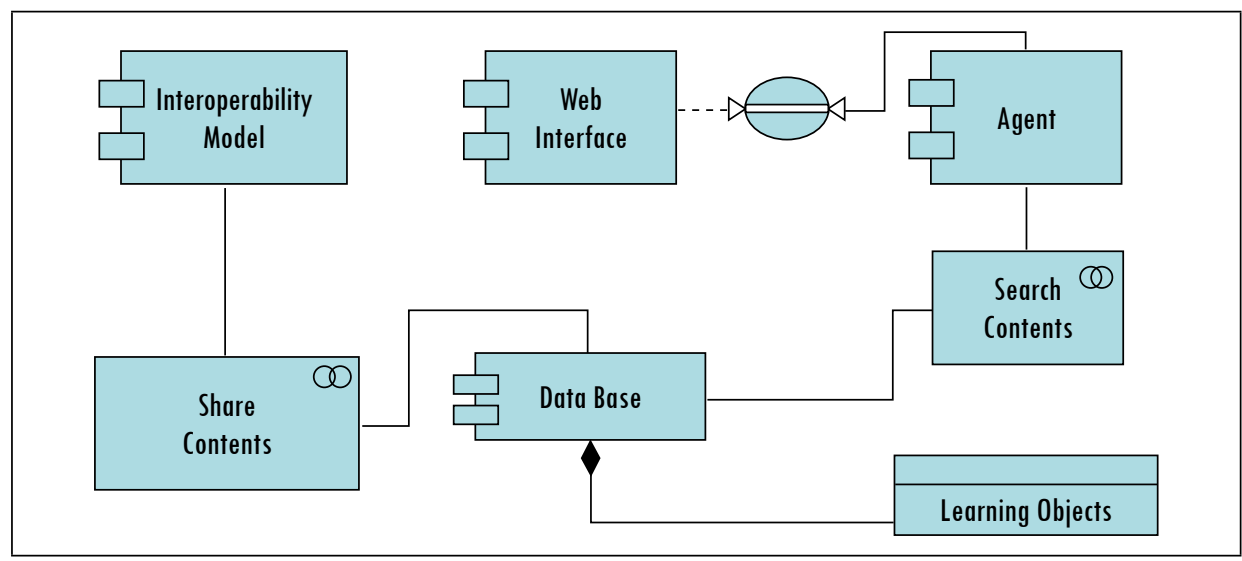

Source: authors' own elaboration

\section{Learning Method and Evaluation}

With the aim of identifying criteria that encourages the usefulness and performance of the developed agent, various aspects have been evaluated through the functionality of the application. The study was developed with a usability expert participation (moderator) and 15 bachelor students from three bachelor careers. Two study methods (individual and focus groups) were used, although all sessions for both methods were carried out with the same protocol to avoid bias on the results. The protocol includes a section of questions using forms based in the Technology Acceptance Model (TAM) through the definition of sixteen questions to asses various aspects about usability and subjective perception of use. Furthermore, it was supplemented with other studies to measure response times associated with the deployment of digital resources during the search process. These studies have been had larger acceptance and dissemination in other work scenarios such as repositories [11], [38], and digital libraries [39], [40], which are the major application areas. Protocol was designed with the aim of obtaining feedback from participants during the focus group assessment sessions and development forms. The protocols used had the intention to assess the effectiveness of the developed agent and the subjective perception of the participants about digital resource search.

In order to carry out this study, a previous short introduction about the developed agent was performed. All users had prior knowledge about search functionalities in each platform (Efront and Moodle). Bearing this in mind, a case study was defined with the aim of assessing the agent in both platforms and the 
conventional search methods that each platform provides. The case study included the search for ten digital resources, previously uploaded and managed through the virtual courses, and lessons created in both platforms. The participants had to perform ten types of searches from three scenarios defined by the moderator: test search, picture search, and videos, through criteria defined by subject, key words, and description. Finally, in accordance with usability factors, every participant had to assess the usefulness of the tool in accordance with the following criteria: usability, navigation, aesthetics, and learnability. At the end of each assessing step, response times were captured for every participant, as requested by the moderator, with the aim of measuring the agent effectiveness in both platforms.

\section{Results}

A summary is presented in Table 1. The results presented show the valuations made by participants about the agent performance based on subjective perceptions.

Table 1. Descriptive statistical analysis for the agent in two LCMS platforms (1: disagree, 5: agree)

\begin{tabular}{|c|c|c|c|c|c|c|c|c|c|c|c|c|}
\hline \multirow[b]{2}{*}{ Efront agent } & \multicolumn{2}{|c|}{1} & \multicolumn{2}{|c|}{2} & \multicolumn{2}{|r|}{3} & \multicolumn{2}{|r|}{4} & \multicolumn{2}{|c|}{5} & \multirow{2}{*}{$\begin{array}{c}\text { Mean } \\
\text { (n) }\end{array}$} & \multirow{2}{*}{$\begin{array}{c}\text { Standard } \\
\text { Deviation (SD) } \\
(\%)\end{array}$} \\
\hline & $\mathrm{n}$ & $(\%)$ & $n$ & $(\%)$ & $\mathrm{n}$ & $(\%)$ & $n$ & $(\%)$ & $\mathrm{N}$ & $(\%)$ & & \\
\hline \multicolumn{13}{|c|}{ Usefulness } \\
\hline Ease of Use & 0 & 0 & 0 & 0 & 3 & 42.8 & 3 & 42.9 & 1 & 14.3 & 3.71 & 0.756 \\
\hline Navigation & 0 & 0 & 0 & 0 & 2 & 28.6 & 4 & 57.1 & 1 & 14.3 & 3.86 & 0.690 \\
\hline \multicolumn{13}{|c|}{ Usability } \\
\hline Learnability & 0 & 0 & 0 & 0 & 2 & 28.6 & 4 & 57.1 & 1 & 14.3 & 3.86 & 0.690 \\
\hline Aesthetic & 0 & 0 & 0 & 0 & 0 & 0 & 3 & 42.9 & 4 & 57.1 & 4.57 & 0.535 \\
\hline \multirow{2}{*}{$\begin{array}{c}\text { Moodle } \\
\text { agent }\end{array}$} & \multicolumn{2}{|c|}{1} & \multicolumn{2}{|c|}{2} & \multicolumn{2}{|r|}{3} & \multicolumn{2}{|r|}{4} & \multicolumn{2}{|c|}{5} & \multirow{2}{*}{$\begin{array}{c}\text { Mean } \\
\text { (n) }\end{array}$} & \multirow{2}{*}{$\begin{array}{c}\text { Standard } \\
\text { Deviation (SD) } \\
(\%)\end{array}$} \\
\hline & $\mathrm{n}$ & $(\%)$ & $n$ & $(\%)$ & $\mathrm{n}$ & $(\%)$ & $\mathrm{n}$ & $(\%)$ & $\mathrm{N}$ & $(\%)$ & & \\
\hline \multicolumn{13}{|c|}{ Usefulness } \\
\hline Ease of Use & 0 & 0 & 0 & 0 & 1 & 12.5 & 5 & 62.5 & 2 & 25.0 & 4.13 & 0.641 \\
\hline Navigation & 0 & 0 & 0 & 0 & 2 & 25.0 & 3 & 37.5 & 3 & 37.5 & 4.13 & 0.835 \\
\hline \multicolumn{13}{|c|}{ Usability } \\
\hline Learnability & 0 & 0 & 0 & 0 & 2 & 25.0 & 5 & 62.5 & 1 & 12.5 & 3.88 & 0.641 \\
\hline Aesthetic & 0 & 0 & 0 & 0 & 0 & 0 & 5 & 62.5 & 3 & 37.5 & 4.38 & 0.512 \\
\hline
\end{tabular}

Source: authors' own elaboration 
The results obtained shows that most of the participants had a positive acceptance criteria to the agent used (ease of use) in the Moodle platform $($ Mean $=4.13 ; \mathrm{SD}=0.641)$ over the Efront platform (Mean=3.71; $\mathrm{SD}=0.756)$. Nevertheless, the results concerning aesthetics show a slight preference in the way Efront deploys results (Mean=4.57; SD=0.535), over the Moodle platform (Mean=4.38; $\mathrm{SD}=0.512)$. Regarding the navigation, most of participants expressed a preference for Moodle (Mean=4.13; $\mathrm{SD}=0.641$ ), over Efront (Mean $=3.86 ; \mathrm{SD}=0.690)$. One of the feasible reasons for these results might be that most of participants indicated through a think aloud session a larger time using Moodle over the Efront platform, which diminished the opportunity to recognize other attractive functionalities about filtering results.

In terms of deployment, most of the participants did not have a clear idea of the methods to filter search processes offered by the tool. This result was shown when they were requested to associate specific resources (text, pictures, and videos). Therefore, a complementary study was defined to identify the effectiveness of the agent in both platforms. In this case, every participant was required to perform ten types of digital resource searches based on the defined criteria in each knowledge area, description, and type of format in each platform. Initially, this process was conventionally developed (without the agent), following a search that was performed using the developed agent. Figure 8 shows the results obtained in both platforms coming from the conventional use defined in each platform and through the developed agent.

In Figure 8, a positive response time to find resources through the use of agents in both platforms is identified. Verifying these results through an statistical analysis, it was identified that samples were homogeneous with a significance level $(\alpha=0.05)$, with the Moodle Agent having the shorter response time $($ Mean $=4.53 ; \mathrm{SD}=0.640)$, over the conventional search method using Moodle (Mean=3.27; $\mathrm{SD}=0.704)$. Likewise, the Efront agent had a shorter response time (Mean $=4.27 ; \mathrm{SD}=0.594)$, in contrast with the obtained results using a conventional searching method on Efront (Mean=3.20; SD =0.862).

Finally, Figure 9 shows a summary of the preferences of the participants to develop search process, in accordance with the obtained results in each search area requested by the moderator. 
Figure 8. Difference between Time Responses of Search Methods

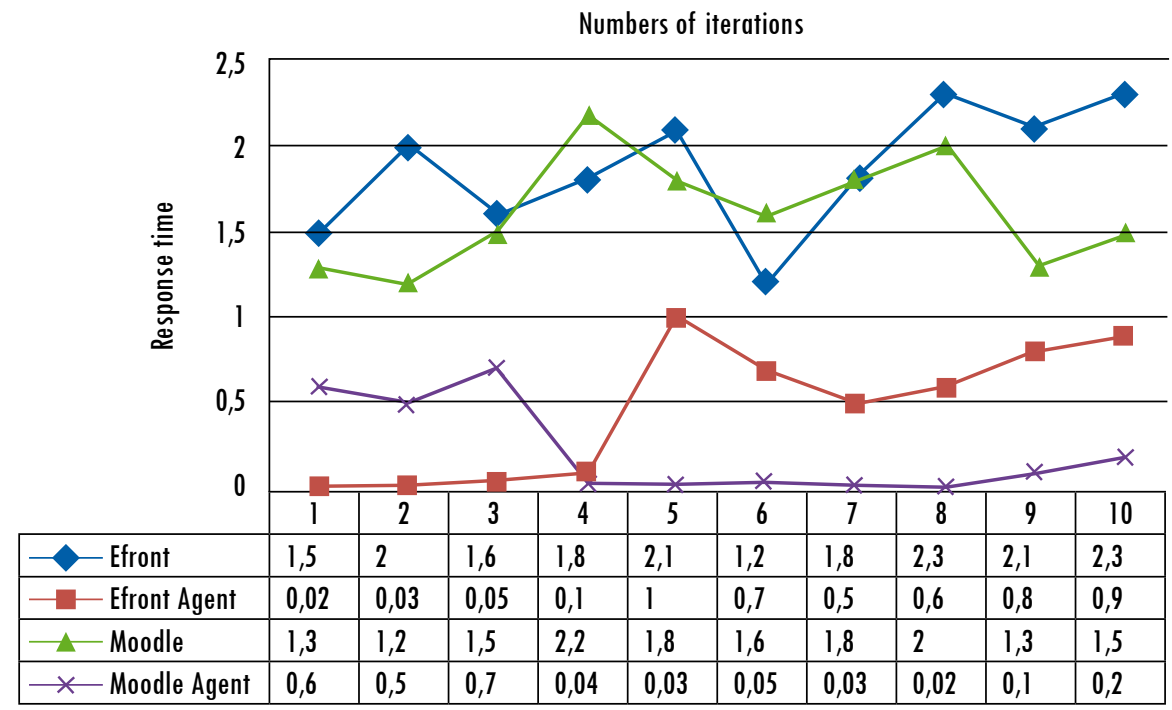

Source: authors' own elaboration

Figure 9. Validation of Search Methods

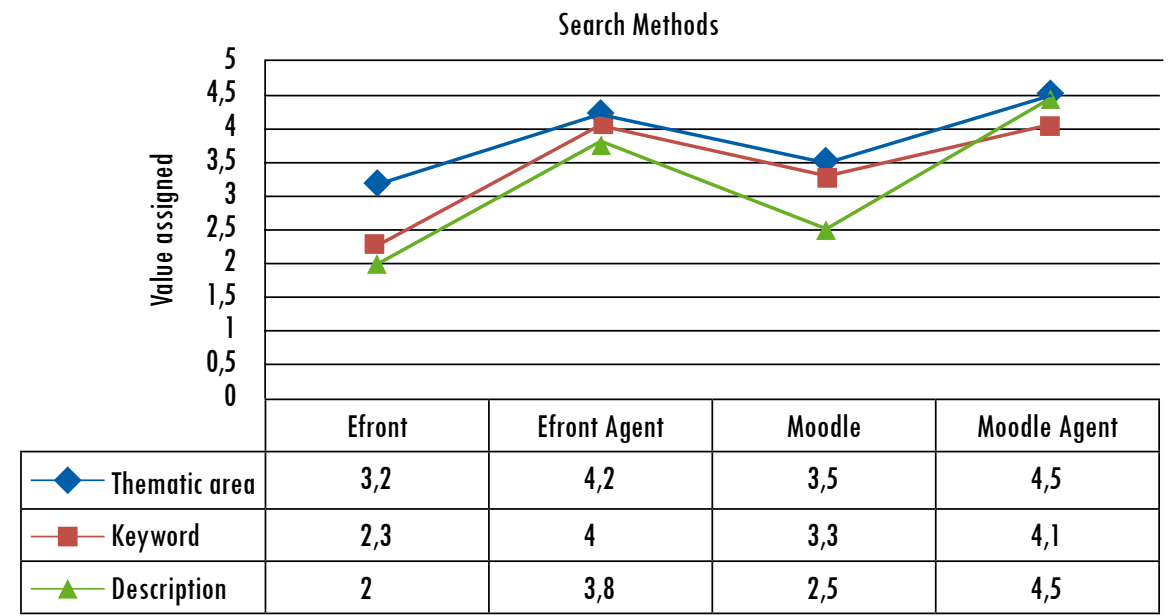

Source: authors' own elaboration 
A remarkable difference is shown in Figure 9 between conventional searches in each platform, in contrast with searches performed using the developed agent. Nevertheless, search preferences on each platform placed the subject criteria as one of the most important in order to develop a search process, being one of the most effective through the use of the developed agent.

\section{Discussion}

For the purpose of applying a communication module in both platforms Efront and Moodle; it was born in mind that the operation mode in each of them has to face the work models to develop a learning process. Considering that the Moodle platform is focused on sharing resources through the courses modality, and teachers or course creators are responsible for carrying out strategies to divulge and disclose contents in other courses, the agent obtains benefits from this work model. These benefits are greater than those provided by Efront, due to the fact that a resource could be used in different courses in diverse manners, which guarantees resource reuse in different work environments. On the other hand, Efront changes its learning process regarding Moodle. Virtual Education; in Efront it is not available in the content but in the lesson; that means a course has one or more lessons, and a lesson has one or more contents. Thereby, the reusable element for Efront is the lesson, enabling a lesson to exist in one or more courses, whenever the teacher or course creator allows it. Hence, the usability factor in each platform is defined by the agent success; others analysis factors are needed for both work approaches which are not included in this study.

Although there are similar studies in order to improve access to learning objects in repositories [37], [41], [42], and the use of the Web of Trust concept [34], [43] there are no studies related with the use of intelligent agents and the use of the Web of Trust in LCMS, such as Moodle and Efront. Notwithstanding the obtained results in the agent response time, it is important to clarify that aspects such as bandwidth connection, number of requests made to the server, and operational burden from other running applications on the server were not considered, elements that significantly affect the agent response time. However, concerning to web services, response time was positive for Moodle over Efront. Nonetheless, the operational model and digital resources stored on each platform might affect their efficiency, therefore, their use should not be excluded in both working models.

It is remarkable to mention that in accordance to the platform, it is important to understand its stored model of metadata. This stored model would enable the 
adaptation process of the agent to perform search activities, and to access learning objects associated to a knowledge area. Therefore, it is necessary to adapt a stored model in each selected platform, with the aim of connecting learning objects in an efficient way towards search processes; this adaptation could take some developing time and management on each one of them.

\section{Conclusions and Future Work}

This proposal provides an effective method to enable interoperability between LCMS platforms with the aim of connecting learning objects. This characteristic facilitates the reuse of contents stored in different LCM platforms, in order to offer relevant information through an academic network. This provides a reliable communication manner, based on the web trust placed between educational institutions that use LCMS platforms.

The confidence relations defined to share learning objects have been identified with emphasis on a centralized architecture, with the aim of enabling any educational institution interested in use of the services to share learning objects with other institutions. The latter service enables a centralized communication to any user who requires access to reliable academic content. Finally, the communication module simplifies the management and control processes, allowing access to a larger quantity of learning objects based on SCORM specifications, similarly to material updating activities to improve their quality.

Due to this working environment, two facets were defined for learning object use. The first facet comes from educational resources supported by SCORM, promoting the use of this specification and encouraging interoperability between different LCMS. The second facet comes from the LOM metadata used to enable search processes and material reuse in diverse LCMS platforms.

In the future, the application of visual search interfaces coming from visual techniques [44]-[47] is expected, with the aim of improving the processes to access relevant learning objects, while it integrates aspects related to the usability of the interfaces [9]. Following this premise, it is important to rely on classification schemes that promote the organization of learning objects according to the specific knowledge area [37]. For this purpose, the representation of knowledge schemes is considered that it could be obtained from ontology and thesaurus, which provide a digital resource classification within a knowledge area.

Furthermore, the association to connection technologies such as Linked data; this type of proposal enables the reuse of educational material stored in LCMS platforms, providing a major educational material scope in a reliable way to 
the community of users. In order to achieve these proposals it is necessary to use knowledge schemes to represent it, such as ontology and thesaurus, that simplify digital content classification, while enriched languages provide learning object localization relevant to the search criteria.

Finally, efforts should be directed toward validating the prototype with the aim of measuring its reliability regarding response time and the relevance of results obtained during the connection of learning objects associated to a knowledge area, which allows the implementation of the method to verify learning objects and trust levels into an academic network.

\section{References}

[1] T. Berners-Lee, "Linked data-the story so far", International Journal on Semantic Web and Information Systems, vol. 5, no. 3, pp. 1-22, 2009.

[2] S. Auer, C. Bizer, G. Kobilarov, J. Lehmann, R. Cyganiak, and Z. Ives, "Dbpedia. A nucleus for a web of open data", in The semantic web, Philadelphia: Springer, 2007, pp. 722-735.

[3] Open Discovery Space (ODS), Open Discovery Space, 2015 [online]. Available: http:// ec.europa.eu/information_society/apps/projects/factsheet/index.cfm?project_ref $=297229$. Accessed Jan. 17, 2015.

[4] M. Fakhimi, L. Stergioulas, et al. "Methodology and evaluation framework for measuring impact of and e-learning platform”, EDULEARN14 Proceedings: 2150-2155, 2014.

[5] Europeana, Europeana professional, 2015 [online]. Available: http://pro.europeana.eu. Accessed Jan. 17, 2015.

[6] B. Haslhofer and A. Isaac, "data.European.eu: The Europeana Linked Open Data Pilot", in Proc. Of the International Conference on Dublin Core and Metadata Applications, Sep 21-23, 2011, The Hague, Netherlands, 2011.

[7] A. Miles, B. Matthews, M. Wilson, and D. Brickley, "SKOS core: simple knowledge organisation for the web" in Proc. at the International Conference on Dublin Core and Metadata Applications "Metadata Vocabularies in Practice", Sep. 12-15, 2005, Leganés, Madrid, Spain.

[8] J. Lacasta, J. Nogueras-Iso, et al., "ThManager. An open source tool for creating and visualizing SKOS”. Information Technology and Libraries, vol. 26, no. 3, pp. 39-51, 2013.

[9] M. Hearst, Search user interfaces. New York, NY: Cambridge University Press, 2009.

[10] M. Khoo, D. Kusunoki, and C. MacDonald, "Finding Problems. When Digital Library Users Act as Usability Evaluators", in Proc. at the System Science (HICSS), 2012 45th Hawaii International Conference on, Jan 4-7, 2012.

[11] H. Kim and Y. Kim, "Usability study of digital institutional repositories", Electronic Library, vol. 26, no. 6, pp. 863-881, 2008. 
[12] LOM, "LOM Standard. Draft Standard for Learning Object Metadata", in IEEE P1484.12/ D4.0, 2003 [online]. Available: http://ltsc.ieee.org/wg 12/20020612-Final-LOM-Draft. html. Accessed Jan. 17, 2015.

[13] SCORM, SCORM, Sharable Content Object Reference Model, 2004 [online]. Available: http:// www.adlnet.org/. Accessed Jan. 17, 2015.

[14] C. Cechinel, S. Sánchez, and M. Sicilia, "Empirical analysis of errors on human-generated learning objects metadata", Communications in Computer and Information Science, vol. 46, pp. 60-70, 2009.

[15] J. Fernández, "Modelos de recuperación de información basados en redes de creencia", tesis doctoral, Universidad de Granada, España, 2001.

[16] J. Pastor, "Diseño de un sistema colaborativo para la creación y gestión de tesauros en Internet basado en SKOS” PhD thesis, 2009.

[17] F. O. Bello-Pérez y J. N. Pérez-Castillo, "Interoperabilidad entre los dominios de la arquitectura, la ingeniería y la construcción y los sistemas de información geográfica1”, Ingeniería y Universidad, vol. 16, no. 1, pp. 183-200, 2012.

[18] M. L. Nelson, "Better interoperability through the open archives initiative", New Review of Information Networking, vol. 7, no. 1, pp. 133-145, 2001.

[19] OAI, The Open Archives Initiative Protocol for Metadata Harvesting, 2002 [online]. Available: http://www.openarchives.org/OAI/2.0/openarchivesprotocol.htm. Accessed Jan. 17, 2015.

[20] X. Bullón, "La visibilidad y los recursos educativos. Técnicas de recolección de metadatos mediante OAI-PMH y LOM”, Idea La Mancha: Revista de Educación de Castilla-La Mancha. Toledo, no. 5, pp. 106-113, 2007.

[21] M. Lytras and M. Sicilia, "Where is the value in metadata?", International Journal of Metadata, Semantics and Ontologies, vol. 2, no. 4, pp. 235-241, 2007.

[22] S. Sánchez and M. Sicilia, "Relationships and commitments in learning object metadata", Proc. ITHET 2004 - 5th Int. Conference on Information Technology Based Higher Education and Training. IEEE, 2004.

[23] D. Gašević, D. Djurić, and V. Devedzic, Model driven engineering and ontology development. Berlin: Springer Verlag, 2009.

[24] J. Gero, "Design prototypes. A knowledge representation schema for design", AI Magazine, vol. 11 , no. 4, p. 26, 1990.

[25] M. Genesereth and S. Ketchpel, "Software agents", Commun. ACM, vol. 37, no. 7, pp. 48-53, 1994.

[26] N. R. Jennings, “On agent-based software engineering”, Artificial Intelligence, vo. 117, no. 2, pp. 277-296, 2000.

[27] S. Franklin and A. Graesser, "Is it an Agent, or just a Program? A taxonomy for autonomous agents", in Intelligent Agents III Agent Theories, Architectures, and Languages. Berlin: Springer, 1997, pp. 21-35. 
[28] H. Kreger, "Fulfilling the Web services promise", Commun. ACM, vol. 46, no. 6, pp. 29-ff, 2003. doi: $10.1145 / 777313.777334$

[29] M. Champion, C. Ferris, E. Newcomer, and D. Orchard, Web services architecture, working draft, 2004 [online]. Available: http://www.w3.org/TR/ws-arch/. Accessed Jan 17, 2015.

[30] IMS, IMS Global Learning Consortium Inc. Overview of Specifications, disposable in, 2013 [online]. Available: http://www.imsglobal.org/overview.cfm. Accessed Jan 17, 2015.

[31] J. Golbeck, B. Parsia, and J. Hendler, Trust networks on the semantic web. Berlin: Springer, 2003.

[32] M. Richardson, R. Agrawal, and P. Domingos, "Trust management for the semantic web", in The Semantic Web-ISWC 2003. Berlin: Springer, 2003, pp. 351-368.

[33] P. Gaona-García, J. Soto, and C. Montenegro, "Trust levels definition on virtual learning platforms through semantic languages", International Journal of Interactive Multimedia and Artificial Intelligence, vol. 1, no. 3, 2010.

[34] O. Corcho, M. Fernández-López, A. Gómez-Pérez, and A. López-Cima, "Building legal ontologies with METHONTOLOGY and WebODE", in Law and the Semantic Web. Berlin: Springer, 2005, pp. 142-157.

[35] M. Fernández-López, A. Gómez-Pérez, and N. Juristo, Methontology: from ontological art towards ontological engineering, 1997 [online]. Available: http://aaaipress.org/Papers/Symposia/Spring/1997/SS-97-06/SS97-06-005.pdf

[36] M. Fernández-López and O. Corcho, Ontological Engineering: with examples from the areas of Knowledge Management, e-Commerce and the Semantic Web. Berlin: Springer, 2010.

[37] P. Gaona-García, D. Martín, A. Fermoso, and S. Sánchez, "A usability study of taxonomyvisualization user interfaces in digital repositories", Journal Online Information Review, vol. 38, no. 2, 2014.

[38] G. Tsakonas and C. Papatheodorou, "Exploring usefulness and usability in the evaluation of open access digital libraries", Information Processing $\mathcal{E}$ Management, vol. 44, no. 3, pp. 1234-1250, 2008.

[39] J. Park, "Metadata quality in digital repositories: A survey of the current state of the art", Cataloging E Classification Quarterly, vol. 47, no. 3-4, pp. 213-228, 2009.

[40] J.Y.L. Thong, W. Hong, and K.-Y. Tam, "Understanding user acceptance of digital libraries: What are the roles of interface characteristics, organizational context, and individual differences?", International Journal of Human-Computer Studies, vol. 57, no. 3, pp. 215-242, 2002.

[41] P. Gaona-García, S. Sánchez-Alonso, et al., "Visualization of information. A proposal to improve the search and access to digital resources in repositories”. Ingeniería e Investigación, vol. 34, no. 1, pp. 83-89, 2014.

[42] D. Martin-Moncunill, S. Sánchez Alonso, P. Gaona-García, N. Marianos, "Applying visualization techniques to develop interfaces for educational repositories. The case of 
Organic. Lingua and VOA3R”, 2013 [online]. Available: http://www.researchgate.net/ publication/236877824_Applying_visualization_techniques_to_develop_interfaces_for_ educational_repositories_the_case_of_Organic.Lingua_and_VOA3R

[43] C. E. Montenegro Marín, P.A. Gaona-García, E. E. Gaona García,., "Plataforma de seguridad basado en autenticidad de contenidos sobre conjunto de especificaciones SCORM". Revista Ingeniería y Competitividad, vol. 12, no. 2, pp. 51-68, 2011.

[44] G. Draper, Y. Livnat, and R. Riesenfeld, "A survey of radial methods for information visualization", Visualization and Computer Graphics, IEEE Transactions on, vol. 15, no. 5, pp. 759-776, 2009. doi: 10.1109/tvcg.2009.23

[45] M. Gleicher, D. Albers, R. Walker, I. Jusufi, C. D. Hansen, and. J. Roberts, "Visual comparison for information visualization", Information Visualization, vol. 10, no. 4, pp. 289-309, 2011.

[46] M. Graham and J. Kennedy, "A survey of multiple tree visualization", Information Visualization, vol. 9, no. 4, pp. 235-252, 2010.

[47] I. Herman, G. Melancon, and M. S. Marshall, "Graph visualization and navigation in information visualization. A survey", Visualization and Computer Graphics, IEEE Transactions on, vol. 6, no. 1, pp. 24-43, 2000. doi: 10.1109/2945.841119 
